\title{
REFLEXIONES DE UNA PROFESORA DE ENSEÑANZA DE MATEMÁTICAS SOBRE LOS INSTRUMENTOS DE EVALUACIÓN
}

\author{
REFLECTIONS OF A MATHEMATICS TEACHING TEACHER ON ASSESSMENT INSTRUMENTS \\ REFLEXÕES DE UMA PROFESSORA DE MATEMÁTICA SOBRE INSTRUMENTOS DE \\ AVALIAÇÃO
}

Maria Jose Costa dos Santos ${ }^{1}$, Vladiana Costa dos Santos²

\section{RESUMEN}

https://doi.org/10.47820/recima21.v3i2.1109

Este texto tiene como objetivo presentar los resultados de una investigación que analizó la evaluación del aprendizaje en el aula a partir de los discursos discentes. En esta investigación los estudiantes de la disciplina de enseñanza de matemáticas, en la carrera de pedagogía de una Institución de Enseñanza Superior-IES, en la ciudad de Fortaleza- Ceará. En el análisis de los instrumentos evaluativos -la prueba didáctica (examen), se utilizó diario de campo (en las observaciones de los discentes y docentes) y cuestionario (en los análisis de los instrumentos evaluativos - la prueba didáctica (examen). Constatamos que los alumnos, agregan a la evaluación en el contexto del aula, el sentido de herramienta de poder para aprobación o reprobación, y presentaron aversión al examen. Por lo tanto, entendemos esto como aspecto revelador y, en ese sentido, deseamos promover un debate sobre cómo, y por qué evaluar, y el sentido formativo que la evaluación debería tener en el contexto del aula.

PALABRAS CLAVE: Examen. Enseñanza de Matemáticas. Estudiantes. Sentidos.

\begin{abstract}
This text aims to present the results of an investigation that analyzed the evaluation of learning in the classroom from the student discourses. Participants in this research were students of the Mathematics Teaching discipline, in the Pedagogy Course of a Higher Education Institution-IES, in the city of Fortaleza-Ceará. In the application, the field diary (in the students' and teacher's observations) and the questionnaire were used (in the analysis of the evaluation instruments - the didactic test (exam). Therefore, we understand this as a revealing aspect and, in this sense, we wish to promote a debate about how, for what and why to evaluate, and the formative sense that the evaluation should have in the context of the classroom.
\end{abstract}

KEYWORDS: Test. Mathematics Teaching. Students. Senses.

\section{RESUMO}

O objetivo deste texto é apresentar os resultados de uma investigação que analisou a avaliação da aprendizagem em sala de aula a partir dos discursos dos alunos. Nesta pesquisa participaram os alunos da disciplina de ensino de matemática do curso de pedagogia de uma Instituição de Ensino Superior-IES, na cidade de Fortaleza-Ceará. $\mathrm{Na}$ análise dos instrumentos avaliativos - a prova didática (exame), foi utilizado um diário de campo (nas observações dos alunos e professores) e um questionário (na análise dos instrumentos avaliativos - a prova didática (exame). Constatamos que os alunos, agregam à avaliação em contexto de sala de aula, o sentido de instrumento de poder para aprovação ou reprovação, e apresentam aversão ao exame. Portanto, entendemos isso como um aspecto revelador e, nesse sentido, queremos promover um debate sobre como e por que avaliar e o sentido formativo que a avaliação deve ter em contexto de sala de aula.

PALAVRAS-CHAVE: Exame. Ensino de Matemática. Alunos. Sentidos.

\footnotetext{
1 Universidade Federal do Ceará

2 Universidade Federal do Ceará
} 


\section{RECIMA21 - REVISTA CIENTÍFICA MULTIDISCIPLINAR ISSN 2675-6218}

REFLEXIONES DE UNA PROFESORA DE ENSEÑANZA DE MATEMÁTICAS SOBRE LOS INSTRUMENTOS DE EVALUACIÓN Maria Jose Costa dos Santos, Vladiana Costa dos Santos

\section{INTRODUCCIÓN}

En Brasil, las políticas públicas de educación, en lo que se refiere al sistema de evaluación a gran escala, han presentado resultados en los índices de evaluación, en gran parte, negativos, y esto se debe a que la evaluación, en general, se relaciona mucho más con la reprobación que con la aprobación, configurándose en un ritual pedagógico, que presenta como papel principal del profesor un tipo de evaluación que pretende evaluar para premiar o castigar.

Este poder atribuido al profesor, emana de las prácticas cotidianas a partir de la dinámica de las relaciones instituidas en la sociedad que lo legitiman. Estas relaciones de poder, mediadas por las prácticas sociales, producen lo que somos, y determinan nuestros saberes, siendo al mismo tiempo reguladores y regulados.

La educación es también una forma de intervención en la sociedad, pero los profesores a veces son impedidos de exponer sus opiniones o participar en la toma de decisiones en reuniones de coordinación sobre las políticas públicas de educación, y así, si dentro de la sala de aula el profesor es dominante, fuera de ella, él es dominado.

En el día a día escolar, un proceso de evaluación pasa por la dominación, adiestramiento, control y disciplina, sin embargo defendemos que la evaluación sea democrática e inclusiva, a partir de un acto crítico que respete las fragilidades del educando y colabore para que él rehaga el recorrido, para finalmente aprender; y el profesor debe ser el mediador en ese proceso de reinversión en el aprendizaje.

En ese entendimiento, el sentido de evaluación no debe ser el de clasificar, pues la evaluación clasificatoria es antidemocrática y excluyente. Aunque estamos siempre en busca de construir resultados, la evaluación debe ser bien definida y delineada promoviendo acciones que subsidian una enseñanza de calidad, a fin de garantizar un aprendizaje de calidad, a partir de un proyecto pedagógico que concilie proceso y resultado.

\section{METOdOLOGÍA DE INVESTIGACIÓN}

Esta investigación es de naturaleza cualitativa, por demostrar mayor análisis del objeto en cuestión y sus particularidades. Los sujetos en este tipo de investigación, son más libres para apuntar sus puntos de vista sobre el objeto de estudio. Dada la subjetividad de las respuestas el propósito no es contabilizar cantidades, sino obtener mayor comprensión del grupo investigado, en relación al objeto. El enfoque de cuño cualitativo trata los datos visando el significado, y tiene como base la percepción del dato en su contexto. Así, utilizamos la descripción cualitativa para captar no sólo la apariencia del fenómeno, sino también sus esencias, buscando explicar su origen, relaciones y cambios, e intentando intuir las consecuencias. (GIL, 1999).

Pretendemos presentar aquí, cuál es el significado del acto de ser evaluado para los estudiantes matriculados en el séptimo semestre del curso de pedagogía, en la disciplina de la 


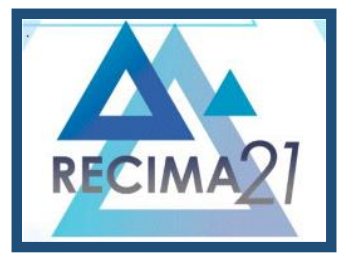

\title{
RECIMA21 - REVISTA CIENTÍFICA MULTIDISCIPLINAR ISSN 2675-6218
}

REFLEXIONES DE UNA PROFESORA DE ENSEÑANZA DE MATEMÁTICAS SOBRE LOS INSTRUMENTOS DE EVALUACIÓN Maria Jose Costa dos Santos, Vladiana Costa dos Santos

enseñanza de las matemáticas, considerando las categorías: (a) castigar o premiar y (b) el proceso y el resultado. Para la recolección y análisis de datos, usamos respectivamente, en la recolección: (a) el diario de campo; (b) cuestionario; y, (c) producción textual. Para los análisis, utilizamos: (a) observaciones de las actividades en el aula; y (b) anotaciones en el diario de campo; y (c) las respuestas al cuestionario.

Para subsidiar la investigación realizamos lecturas de artículos publicados en actas de eventos internacionales y nacionales, libros con comité editorial, además de banco de datos virtuales de periódicos con Qualis ${ }^{1 /}$ Capes $^{2}$.

Nuestro problema se sustenta en el supuesto de que el examen de matemáticas, en el caso de los alumnos del curso de Pedagogía, siempre es motivo de rechazo, y de ese modo, pretendemos desmitificar ese sentido de evaluación, y para tanto, dos cuestiones fueron básicas: (a) ¿Cuál es el problema? la relación de los alumnos de la disciplina de enseñanza de matemáticas con el examen de Matemáticas ?, y, (b) ¿Es posible evaluar, sin clasificar, utilizando la prueba didáctica?

Este ensayo se compone de esta sección que trata la introducción (presentación y justificación del tema), seguida de otras secciones que apoyan el objeto de estudio (el examen), y las consideraciones y referencias. En la siguiente sección se presenta el marco teórico.

\section{MARCO TEÓRICO: LA EVALUACIÓN EN CLASE DEL CURSO DE FORMACIÓN DOCENTE}

En la obra Vigilar y Punir, Foucault (1987, p. 202) destaca que "En la esencia de todos los sistemas disciplinarios, funciona un pequeño mecanismo penal". El poder disciplinario y las técnicas de vigilancia permean los sistemas escolares y controlan esos espacios por medio de un pacto legitimado socialmente, esto se da "sin duda, al uso de instrumentos simples: la mirada jerárquica, la sanción normalizadora y su combinación en un procedimiento que le es específico, el examen (FOUCAULT, 1987, p. 195).

El autor destaca que,

\begin{abstract}
El examen combina las técnicas de la jerarquía que vigila y las de la sanción que normaliza. Es un control normalizante, una vigilancia que permite calificar, clasificar y castigar. Establece sobre los individuos una visibilidad a través de la cual son diferenciados y sancionados. Es por eso que, en todos los dispositivos de disciplina, el examen es altamente ritualizado. En él se vienen a reunir la ceremonia del poder y la forma de la experiencia, la demostración de la fuerza y el establecimiento de la verdad (FOUCAULT, 1987, p. 209).
\end{abstract}

En este sentido, destacamos que las relaciones microfísicas del poder que ocurren en el ámbito de la escuela, especialmente en el aula, son evidenciadas en el papel del profesor que regula, controla, instruye, educa, adiestra y se convierte en una referencia del proceso de

\footnotetext{
${ }^{1}$ Conjunto de procedimientos utilizados por Capes para estratificación de la calidad de la producción intelectual programas de postgrado, basados en la publicación en revistas científicas de artículos de docentes afiliados a las Instituciones de Educación Superior (IES) brasileñas. (Fuente: http://www.sibi.usp.br/apoiopesquisador/escritura-y texto científico).

${ }^{2}$ Coordinación de Perfeccionamiento de Personal de Nivel Superior. (Fuente: www.capes.gov.br).
} 


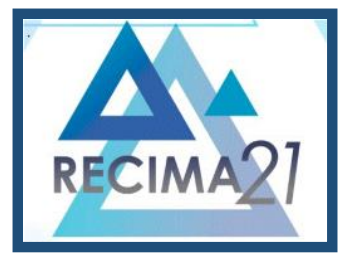

\section{RECIMA21 - REVISTA CIENTÍFICA MULTIDISCIPLINAR ISSN 2675-6218}

REFLEXIONES DE UNA PROFESORA DE ENSEÑANZA DE MATEMÁTICAS SOBRE LOS INSTRUMENTOS DE EVALUACIÓN Maria Jose Costa dos Santos, Vladiana Costa dos Santos

aprendizaje del alumno, es lo que Foucault (1996) denominó panoptismo. Para el autor "(...) el aula formaría un gran cuadro único, con entradas múltiples, bajo la mirada cuidadosamente 'clasificadora' del profesor (FOUCAULT, 1987, p. 173).

Luckesi (2008, p. 102) dice que la evaluación, sin objetivo de construcción de aprendizaje, parece más fantasía, o acciones inofensivas y aleatorias, sin meta, la evaluación se transforma en mero activismo. Para el autor, la evaluación, a diferencia de la verificación, es un acto que supera el deseo de apenas evaluar, pero exige del profesor, una toma de decisión de qué hacer con el resultado de esa acción. Y aún, en ese proceso, debe considerar que el alumno, al errar, "el error no debe añadir culpa y castigo." (LUCKESI, 2008, p. 59), y si debe ser objeto de reinversión en el proceso de aprendizaje.

Defendemos una evaluación formativa, diagnóstica y continua que posibilite a la escuela el desarrollo de una educación más comprometida con la transformación social, la que se preocupe más por el proceso que por el resultado, que promueva la mejora de la educación, y elimine la idea de evaluar para medir y castigar.

Una evaluación de este tipo, rompe con el discurso de una educación bancaria (FREIRE, 1996), abriendo espacio para una enseñanza innovadora y creativa, pues ayuda en la identificación de los conocimientos previos de los estudiantes, facilitando al profesor presentar un pronóstico del aprendizaje.

Con ello, se sustituye el acto de castigar por el de educar, por el acto en que alumno y profesor conversan, y hay un quiebre de paradigma de evaluar para castigar o premiar, presentando el real sentido de la evaluación que es el del profesor auxiliar en la construcción del aprendizaje del alumno de forma concreta; propiciando así la relación profesor-contenido-alumno, también para este proceso de construcción de un aprendizaje de calidad. En este sentido, destacamos a continuación la figura 1, que presenta el triángulo instruccional, el cual objetiva la interacción profesor - alumno contenido.

Figura 1. Triángulo Instruccional

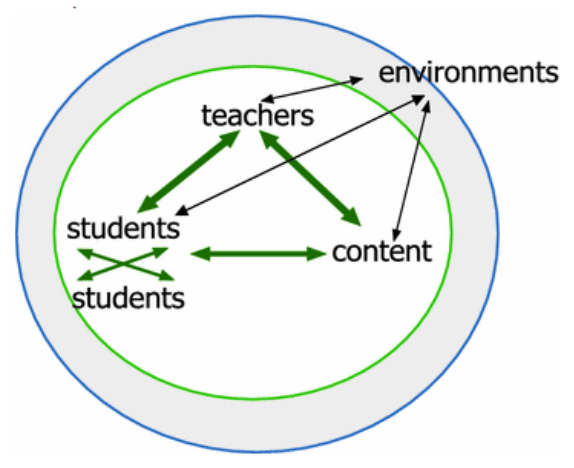

Fuente: ICME ${ }^{3}$ (2013, p. 820)

\footnotetext{
${ }^{3}$ Ver Ball e Forzani (2007) para una discusión de cómo este triángulo instructivo se relaciona y difiere de otros usos de "triángulos" para representar la enseñanza y el aprendizaje.
} 


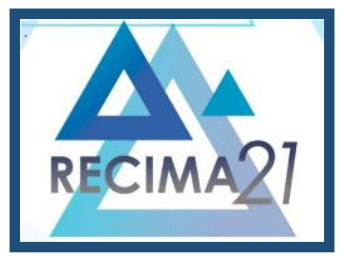

\section{RECIMA21 - REVISTA CIENTÍFICA MULTIDISCIPLINAR ISSN 2675-6218}

Traemos para discusión esta idea Ball (2007), porque estamos de acuerdo en que el aprendizaje no ocurre por casualidad en aulas, se hace necesario un trabajo de enseñar lo más habilidoso cuanto pueda ser, de lo contrario no habrá aprendizaje, y el trabajo del profesor será puesto en riesgo, basta ver los resultados de las mismas evaluaciones.

\subsection{TIPOS DE EVALUACIÓN}

La evaluación del tipo formativo es toda y cualquier evaluación que considera a los estudiantes como seres distintos, y el ambiente del aula lleno de diversidades comportamentales. Este tipo de evaluación, en consecuencia, obliga al docente a tomar una actitud ante el resultado que el alumno presentó, no es sólo decir que se equivocó, sino por qué se equivocó. Hay un compromiso pedagógico del profesor con ese tipo de evaluación. Conjuntamente la evaluación diagnóstica, la evaluación formativa posibilita que el profesor transforme el ambiente de aula y sus clases a partir de las necesidades y realidades de los estudiantes.

La evaluación formativa es una estrategia pedagógica de lucha contra fracasos educativos y diferencias de aprendizaje. Para ello, es importante el docente en el desarrollo de una secuencia didáctica, detectar el camino ya recorrido por el alumno en relación al aprendizaje del género y lo que aún falta por recorrer, para hacer intervenciones didácticas acertadas (GONÇALVES; NASCIMENTO, 2010, p. 244).

Entendemos que la evaluación diagnóstica subvenciona la evaluación formativa, y la evaluación formativa fundamenta la evaluación diagnóstica. Una visando la identificación de los conocimientos previos de los estudiantes, la otra buscando de posesión de esos conocimientos trabajando la diversidad con fines formativos.

En este sentido, Mendez (2002) resalta que la evaluación del aprendizaje en el aula debe ser formativa, objetivando siempre la mejora de la práctica al servicio de los profesores y alumnos, de modo continuo e integrado al currículo. El autor todavía destaca que la evaluación dentro de ese continuum asume características procesales y continúa considerando el currículo y el aprendizaje. En cuanto a esta perspectiva de evaluación, Luckesi (2008) dice que la evaluación de naturaleza clasificatoria, sólo es eficiente para la domesticación de los alumnos, y en ese sentido, es necesario que la escuela, como un instrumento dialéctico para no ser autoritaria y conservadora, desempeña la función diagnóstica de la evaluación.

Y así, el proceso de diagnosticar para formar, ocurre dentro de un continuum que destacamos en la figura 2 a continuación. 


\section{RECIMA21 - REVISTA CIENTÍFICA MULTIDISCIPLINAR ISSN 2675-6218}

REFLEXIONES DE UNA PROFESORA DE ENSEÑANZA DE MATEMÁTICAS SOBRE LOS INSTRUMENTOS DE EVALUACIÓN Maria Jose Costa dos Santos, Vladiana Costa dos Santos

Figura 2. Triangulación de la evaluación del aprendizaje.

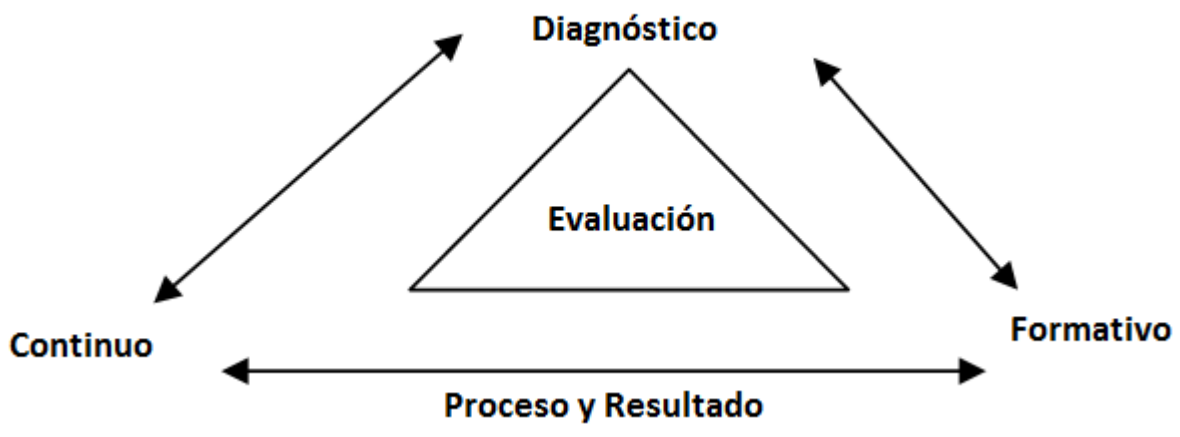

Fuente: Elaboración propia.

El profesor, en ese recorrido, necesita tener compromiso pedagógico y buscar desprenderse de la concepción de evaluación instituida por la sociedad y por las políticas educativas, pues éstas son sobre todo clasificatorias y excluyentes.

\subsection{INSTRUMENTOS EVALUATIVOS UTILIZADOS EN LA DISCIPLINA DE ENSEÑANZA DE MATEMÁTICAS}

En cuanto al análisis de los instrumentos evaluativos utilizados en la disciplina de enseñanza de matemáticas, nos importa en particular el examen, y la relación de poder de ese instrumento sobre el aprendizaje de los estudiantes, a partir de sus discursos.

Las concepciones sobre evaluación de Luckesi (2008), Vasconcellos (2007), Perrenoud (1999) y Hoffman (2005) nos ayudan específicamente, en la diferenciación y clarificación del verdadero sentido de la evaluación del aprendizaje en el aula.

En general, la evaluación se resume en que "medida" el otro aprendió, cuánto de cero a diez tiene conocimiento, y eso refleja cuanto los instrumentos de evaluación están vinculados a los resultados, y de ese modo, modificar el proceso se convierte complejo. Históricamente, Perrenoud (1999) destaca que el verdadero propósito de evaluar ya llegó en la educación de forma distorsionada, y con la finalidad de en gran parte seleccionar, excluir, marginar.

Este tipo de práctica evaluativa para Vasconcellos (2007) lleva a los alumnos, simplemente a olvidar casi todo aquello que respondieron en el examen. En consecuencia, esta idea de evaluación para clasificar induce a los estudiantes a reaccionar de forma negativa a este tipo de instrumento.

De hecho, la palabra evaluar provoca muchas reflexiones y ponderaciones didácticas. La Ley oㅜ 9394/96 ley de directrices y bases de la educación nacional-LDBEM (BRASIL, 1996), establece que la evaluación debe ser continua y acumulativa del desempeño del estudiante, con prevalencia de los aspectos cualitativos sobre los cuantitativos y de los resultados durante el período de las eventuales 


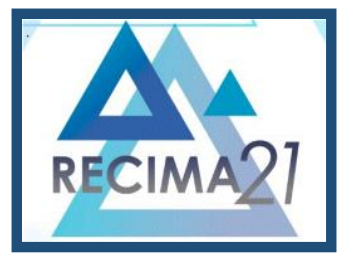

\title{
RECIMA21 - REVISTA CIENTÍFICA MULTIDISCIPLINAR ISSN 2675-6218
}

REFLEXIONES DE UNA PROFESORA DE ENSEÑANZA DE MATEMÁTICAS SOBRE LOS INSTRUMENTOS DE EVALUACIÓN Maria Jose Costa dos Santos, Vladiana Costa dos Santos

pruebas finales. Los parámetros curriculares nacionales de las matemáticas-PCNM (BRASIL, 1997), señalan que

\begin{abstract}
La evaluación forma parte del proceso de enseñanza y aprendizaje. Se centra en una gran variedad de aspectos relativos al desempeño de los alumnos, como la adquisición de conceptos, el dominio de los procedimientos y el desarrollo de actitudes. Pero también deben ser evaluados aspectos como la selección y dimensionamiento de los contenidos, prácticas pedagógicas, condiciones en que se procesa el trabajo escolar y las propias formas de evaluación (BRASIL, 1997, p. 20-21).
\end{abstract}

Cuando se trata de evaluar existe una preocupación por parte del evaluador sobre cuales prácticas pedagógicas son más adecuadas y qué instrumento dialoga con esa práctica. Para Valente (2008) evaluar el aprendizaje matemático, es una tarea compleja que exige mirar no sólo para el resultado, el objetivo del examen, sino para el proceso, como destaca el PCNM (BRASIL, 1997). Así, concordamos con Freire (1987) cuando no se pone en contra de la evaluación, pero entiende que los métodos de evaluación deben servir para formar ciudadanos críticos y no silenciarlos.

Y destaca,

\begin{abstract}
La cuestión que se plantea a nosotros, como profesores y alumnos críticos y amorosos de la libertad, no es, naturalmente estar en contra de la evaluación, pues es necesaria, sino resistir a los métodos silenciadores con los que a veces es realizada. La cuestión que se plantea a nosotros es luchar en favor de la comprensión y de la práctica de la evaluación como instrumento de apreciación, que hacer de sujetos críticos al servicio, de la liberación y no de la domesticación (FREIRE, 1987, p. 72).
\end{abstract}

En el curso de Pedagogía que analizamos, la elección de la realización del examen como herramienta de evaluación, no es tan común, pues otros métodos evaluativos son escogidos, tales como: seminarios, talleres, producción de artículos, etc. y tal vez tal cultura aleja a los estudiantes de ese tipo de instrumento de evaluación.

También vale resaltar, los testimonios de malas experiencias que ellos tuvieron aún en la educación básica, esa relación, en parte es más afectada cuando se trata de las matemáticas. Esto aparece más fuertemente durante la observación de una de las clases de enseñanza de matemáticas, cuando una alumna revela: "odio matemáticas con todas mis fuerzas".

En las matemáticas, el acto pedagógico de evaluar comprende algunos elementos: (a) la interpretación del alumno acerca de la situación-problema; (b) las estrategias utilizadas para la solución; (c) conocimientos en juego; y (d) la formalización matemática. Estos elementos se revelan más claramente, durante la realización del examen, cuando el alumno se enfrenta a las situaciones problemáticas propuestas, y él inmediatamente se refiere al profesor para que éste le explique lo que el problema pide.

Esto tiene graves implicaciones para el resultado del alumno en el examen, pues es importante que él desarrolle el razonamiento, su pensamiento matemático a partir de la 


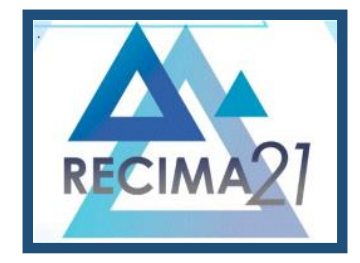

\section{RECIMA21 - REVISTA CIENTÍFICA MULTIDISCIPLINAR ISSN 2675-6218}

interpretación que la subjetividad del examen le impone. En ese momento, el alumno puede pensar: 'si yo no respondo correctamente, ¿seré rechazado?

El "miedo" de no ser bien evaluado en el examen puede caracterizarse en un obstáculo para obtener éxito. Este es el sentimiento que los estudiantes quieren que los profesores perciban. Sin embargo, el profesor, a veces, en demostración de poder, elabora un método de evaluación incoherente con su práctica pedagógica en el aula, y sobre eso Freire (1987, p. 89) alerta "que no es cierto, sobre todo del punto de vista democrática, que se seré mejor profesor cuanto más severo, más frío, más distante y "gris" me ponga en mis relaciones con los alumnos, en el trato de los objetos cognoscibles que debo enseñar / evaluar".

Cabe recordar que la evaluación debe ser democrática en el sentido que no rotula, pero proporciona apropiación y acercamiento mayor del estudiante, sea él niño, joven o adulto, con el saber en juego; y debe posibilitar al educando y al educador la re significación de los procesos de enseñanza y de aprendizaje, provocada por una evaluación con discusiones relacionadas a la práctica pedagógica que deja de ser un momento terminal del proceso educativo para transformarse en oportunidades de aprendizaje.

En esta trayectoria, el profesor tiene el deber de entender que una de las funciones de la evaluación es ofrecerle información sobre los procesos de enseñanza y de aprendizaje, posibilitando a partir de los resultados reorientar su práctica, Hoffmann (2005, p. 15) advierte que "un maestro que no evalúa constantemente la acción educativa, en el sentido de indagación e investigación del término, instala su enseñanza en verdades absolutas, prefabricadas y terminales.

También destaca que la evaluación es inherente e indisociable a la educación cuando se concibe a partir de una problematización y reflexión sobre la acción fundada en un proyecto pedagógico, que asiste en la elección adecuada de instrumentos evaluativos para esta práctica (HOFFMANN, 2005).

Es sobre los instrumentos de evaluación, que tratamos a continuación.

\section{SENTIDO DE EVALUACIÓN EN LA DISCIPLINA DE ENSEÑANZA DE MATEMÁTICAS}

En el núcleo de las relaciones microfísicas del poder, analizamos un aula de un curso superior, de una IES pública del estado Ceará, en que los estudiantes frente a los instrumentos de evaluación, discrepaban del método empleado, en especial, presentaban aversión al uso del examen.

Los estudiantes que participaron en la investigación, estaban regularmente matriculados en la disciplina obligatoria de enseñanza de matemáticas, en el semestre 2017.1, cuya carga horaria es de 96h / a o 6 créditos, ese es obligatoriamente el tiempo didáctico para trabajar en el aula los contenidos de los bloques indicados en los Parámetros Curriculares Nacionales de Matemáticas PCNM (BRASIL, 1997), son ellos: Números y operaciones, Espacio y Forma, Magnitudes y 


\section{RECIMA21 - REVISTA CIENTÍFICA MULTIDISCIPLINAR ISSN 2675-6218}

REFLEXIONES DE UNA PROFESORA DE ENSEÑANZA DE MATEMÁTICAS SOBRE LOS INSTRUMENTOS DE EVALUACIÓN Maria Jose Costa dos Santos, Vladiana Costa dos Santos

Medidas, Tratamiento de la Información, además de metodologías de enseñanza y teorías del aprendizaje.

Con toda esa relación de contenidos a ser trabajada, el profesor acaba por acelerar la "enseñanza" en detrimento del "aprendizaje", pues precisa "entregar" mínimamente el menú de la disciplina. En este sentido, los alumnos pasan a hacer críticas, en parte por las lagunas que traen del contenido desde la formación de las matemáticas en la educación básica, y en parte por ser cobradas en un espacio restringido de tiempo por cuenta de los procesos de evaluación que la profesora propone.

En el inicio de la disciplina, la profesora presenta el plan de enseñanza, que se refiere a la disciplina y la metodología de evaluación, y así realiza el acuerdo didáctico (SOUSA, 2015). En ese momento, la metodología es debidamente esclarecida con la finalidad de explicar a todos sobre los procedimientos adoptados para el desarrollo de las clases de la disciplina de enseñanza de matemáticas.

En el primer día de clase, se presenta el plan de enseñanza y en detalle los contenidos a ser trabajados durante el semestre. En el plano de enseñanza, la profesora propone: (a) aula expositiva-dialogada; (b) dinámicas de grupos; (lecturas y estudios de textos y síntesis en grupos, investigaciones orientadas), (c) talleres pedagógicos; (uso de materiales analógicos y digitales, (QVL, Tangran, poliedros, objetos de aprendizaje), (d) asistir, discutir y analizar video-clases y software.

Las actividades se orientan en sala y tienen como presupuestos metodológicos los principios de la metodología de enseñanza, investigación y formación Secuencia Fedathi (SANTOS, 2016), así como, en las concepciones teóricas de las tendencias en educación matemática.

Es responsabilidad de los discentes ante las actividades, y en atención al acuerdo didáctico firmado con el profesor, realizar: (i) lecturas de los textos; (ii) participación en los debates en la sala; (iii) informes de las clases; (iv) la producción de un artículo; (v) observar una clase de matemáticas en la escuela; y, finalmente, (vi) la prueba didáctica. Cómo el alumno será evaluado, es la mayor preocupación de ellos y, por lo tanto, es importante estar bien definido desde el primer día de clase, con el acuerdo didáctico. La relación profesor y alumno debe ser la más transparente posible.

Entre las propuestas de instrumentos evaluativos como informe de las clases, participación en sala, producción de artículo, observación de una clase de matemáticas en la escuela, el instrumento más cuestionado es el examen; y el hecho de ser el único instrumento individual y sin consultas a materiales de clase (libro, cuaderno, textos, etc.), causa trastornos en la sala y sacude la relación profesor - alumno, aflorando las relaciones de poder.

El Examen es siempre un instrumento de evaluación que preocupa al estudiante, pues para él, hay un impacto directamente en su nota final, sin embargo, para el profesor ese instrumento presenta posibilidades de comparación de las expectativas iniciales con los resultados alcanzados, o sea, desde donde se partió y donde se desea llegar, considerando más el recorrido, y menos el resultado. 


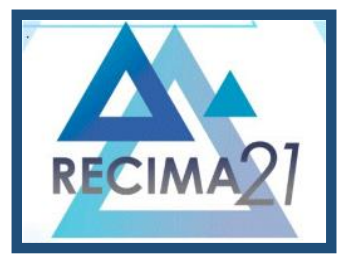

\section{RECIMA21 - REVISTA CIENTÍFICA MULTIDISCIPLINAR ISSN 2675-6218}

Los instrumentos evaluativos utilizados en la disciplina de enseñanza de matemáticas, son propuestos con la finalidad de diagnosticar los conocimientos adquiridos por los discentes, durante el proceso formativo, y a partir de ese diagnóstico planificar mejor las acciones pedagógicas, y, consecuentemente, reorientar la elección de la herramienta de evaluación, considerando que debe ser apropiada: (a) al tipo de comportamiento y de habilidad a ser evaluadas; (b) a los contenidos trabajados en el proceso de enseñanza; y, c) al lenguaje y la precisión de la comunicación. Con esta explicación, aunque hemos presentado las diversas formas de evaluación ejercidas en la disciplina, destacamos a continuación el uso del examen en la disciplina de enseñanza de matemáticas, por ser la herramienta de evaluación más cuestionada por los alumnos.

\section{ANÁLISIS DE RESULTADOS: EL EXAMEN}

Durante la disciplina de Enseñanza de Matemáticas, constatamos que el examen se realiza en tres momentos a lo largo del semestre. Como se dijo anteriormente, se utilizan varios instrumentos de evaluación, pero para la profesora de la disciplina, a pesar de que el examen es el instrumento de mayor rechazo, también es el de mayor impacto en la formación de los alumnos, pues los saca de la zona de confort y los coloca en posición de desafío.

Para la profesora de la disciplina,

"Es en ese momento que ellos paran para pensar más fuertemente sobre las clases de la disciplina, y les permite reflexionar sobre cómo sus alumnos se sentirán ante ese instrumento, y pueden decidir si van a querer usarlo o no, para qué, y por qué. "(DISCURSO DE LA PROFESORA)

En la disciplina de enseñanza de matemáticas, el Examen representa una de las notas parciales de un total de tres notas. En el examen, la profesora opta por dividirlo en tres partes (al final de las unidades de enseñanza), y esas partes al final del semestre se suman, generando una nota. Dos de las partes de la prueba tienen tres cuestiones y otra parte de la prueba tiene cuatro cuestiones, en un total de 10 preguntas y cada una de las cuestiones vale 1 punto. Cada parte del examen tiene fecha definida en el calendario de la asignatura, y toda la información se presenta al alumno en el primer día de clase.

La forma en que las preguntas se presentan, varían según el contenido y la forma en que se debatió en la sala. Cuando las clases son más exitosas, las preguntas son más difíciles, cuando la clase demuestra menos intimidad con los contenidos, las preguntas son más simples (simples no desde el punto de vista fácil, pero adecuada al debate en el aula), es decir, cuanto más el alumno demuestre envolvimiento con el contenido, la profesora tiene condiciones de elaborar mejor el examen, exigiendo mayor complejidad en la interpretación de las cuestiones.

Para nosotros el examen se constituye en uno de los instrumentos evaluativos importantes de los procesos de enseñanza y de aprendizaje, y permite al profesor evaluar al alumnado por sus diversas posibilidades. 


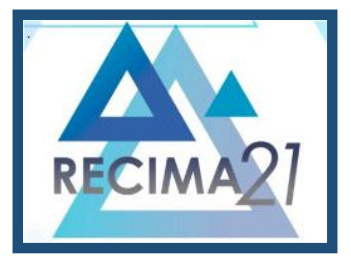

\section{RECIMA21 - REVISTA CIENTÍFICA MULTIDISCIPLINAR ISSN 2675-6218}

Con esta reflexión, el examen: (1) permite la reflexión del estudiante, así como la organización de la información, opiniones, puntos de vista, los conceptos y el conocimiento; (2) excluye el factor suerte y adivinación; (3) no anula cierta subjetividad de juicio, porque las producciones son más libres y no se puede aplicar patrón rígido de corrección; (4) retiene vestigios personales (letra, estilo), siendo de una extensión considerable para corrección; (5) Posibilidad de evaluar la capacidad de organización, análisis y aplicación de contenidos; (6) las palabras de comando (comente, argumente, justifique, caracterice, identifique, lo que usted sabe sobre) deben ser utilizadas en el texto y en el contexto permitiendo el parámetro correcto para la cuestión. (ZANON; ALTHAUS, 2008, p. 8-9).

A pesar de que el examen es visto por los alumnos como instrumento de exclusión, para la profesora de la disciplina enseñanza de matemáticas, ella es un instrumento de formación. Por lo tanto, es a partir de esa concepción que vamos a entender la relación de los estudiantes con ese instrumento.

En las clases de la disciplina de enseñanza de matemáticas, se observaron 27 estudiantes, pero después de los análisis vimos que los testimonios se asemejan, y por lo tanto, para no parecer repetitivos, presentamos algunos de los testimonios, como del alumno (A), sobre el Examen

"Creo que la prueba (el Examen) puede ser uno de los instrumentos, pero no el único o el preponderante, porque la presión de una evaluación hace que a menudo los alumnos olviden los contenidos por nerviosismo, miedo o presión". (ALUNO A)

Se percibe que la aversión del estudiante al instrumento revela que para él el examen reprueba, y sus sentimientos en relación al instrumento son de miedo, nerviosismo y presión; y en nada coincide con el sentimiento que la profesora expresa, pues ella declaró en sus clases que el examen era un instrumento que permitía al estudiante ser evaluado de una manera más personalizada, pero que primaba observar la evolución del estudiante en el aprendizaje, y la posibilidad de repensar su práctica en el aula. En esto observamos que para la profesora evaluar es un proceso y no un resultado

Pero lo contradictorio siempre imperaba, y el estudiante B, refuerza,

"No considero como un instrumento que demuestre efectivamente el aprendizaje adquirido durante el semestre." (ALUMNO B)

Observamos que en algún momento la profesora quiso presentar el examen como instrumento de medición del aprendizaje, pero en vez de eso, siempre reforzaba la idea de que el examen proporcionaba un momento ideal de reflexión sobre conceptos trabajados en las clases; y que la situación de trabajar sin investigación en los materiales, o con los colegas, posibilitaba al propio estudiante la autoconfianza en su saber. Pero el alumno $\mathrm{C}$, enfatiza, 


\section{RECIMA21 - REVISTA CIENTÍFICA MULTIDISCIPLINAR ISSN 2675-6218}

REFLEXIONES DE UNA PROFESORA DE ENSEÑANZA DE MATEMÁTICAS SOBRE LOS INSTRUMENTOS DE EVALUACIÓN Maria Jose Costa dos Santos, Vladiana Costa dos Santos

"Hay una presión con respecto a este instrumento de evaluación." (ALUMNO C)

Esta presión reflejada en los sentidos de los estudiantes es muy particular de ellos, pues las evaluaciones eran presentadas al inicio de la disciplina por la profesora, señalados todos los contenidos a ser abordados, y como el examen no era la única forma de evaluación, en este caso para profesora, los estudiantes querían evidenciar en el aula, la relación de poder, colocando al profesor en situación de dominante y ellos como dominados.

Para la profesora, el alumno que ve la prueba como instrumento opresor, tiene serios problemas de comprensión del sentido de lo que es evaluar, pues trae en sí la concepción de una evaluación que durante mucho tiempo lo excluyó del proceso. Perrenoud (1998) dice que todas las evaluaciones son formativas desde que contribuyan a la regulación de los aprendizajes, y el autor continúa afirmando que puede ser considerada formativa toda práctica de evaluación continua que pretenda contribuir a mejorar los aprendizajes en curso.

“(...) la prueba (el examen) puede demonstrar el aprendizaje parcialmente (...)" (ALUMNO D)

Fue importante escuchar a la profesora sobre la definición que los estudiantes tienen de las evaluaciones propuestas por ella, pues para ella las evaluaciones no tenían la intención de verificar el aprendizaje como resultado final, y si, buscaba la evolución de los conceptos presentados en sala.

Aunque la profesora dice que no controla el conocimiento en el aula, presenta la necesidad de disciplinar el trabajo pedagógico, y hace ese control a partir de procesos de subjetivación que se originan de las prácticas cotidianas que legitiman sus acciones.

De modo, realista, el discurso de los estudiantes muestra que ellos tienen un sentido negativo sobre la prueba didáctica, y en efecto, eso les impide percibirla como un instrumento de formación, y los imposibilita de ver en la disciplina de enseñanza de matemáticas, que el examen no es el único instrumento por el cual son evaluados durante las clases. Este sentimiento, también les impide percibir las posibilidades que ese instrumento puede agregar en su proceso de aprendizaje, sólo logran ver la prueba didáctica como un medio para obtener una nota que podrá reprobarlos o aprobarlos.

En general, tanto en las observaciones en sala, como en las respuestas a las preguntas de la investigación, fue posible concluir que la mayoría de los alumnos consideran que la prueba puede demostrar su aprendizaje sólo parcialmente, pero para la profesora, el examen es un instrumento de formación privilegiada que si bien elaborado, auxilia al profesor también a repensar su práctica.

Nuestro sentido de evaluación comprende que el valor de la evaluación no está en el instrumento en sí, sino en el uso que se hace de él. Así, en lo que se refiere al examen, uno de los instrumentos evaluativos de la disciplina de enseñanza de matemáticas, es muy probable que en el 


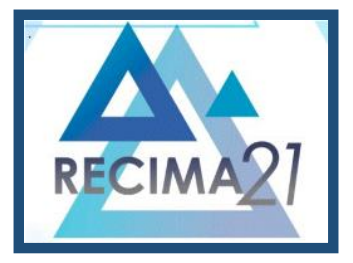

\section{RECIMA21 - REVISTA CIENTÍFICA MULTIDISCIPLINAR ISSN 2675-6218}

futuro, los alumnos perciban su importancia y pasen a utilizarlo, siempre teniendo en cuenta la realidad y las soluciones a situaciones desafiantes que se presentan en lo cotidiano del aula, observando: (a) enunciado; (b) tipo de preguntas; y, (c) la realidad del alumno.

A partir de las reflexiones sobre el discurso de los estudiantes, y el feedback de la profesora, vimos que tal vez, en la escuela, el poder del profesor en sala, es más legitimado, pues se trata de un adulto 'dominando' a un niño, pero en la academia, los estudiantes que ya son adultos, no son como trata Foucault (1987) "cuerpos dóciles", es decir, cuerpos obedientes y "buenos".

El alumno adulto, reclama, protesta y el profesor frente a esa resistencia, toma la decisión si va a ceder o imponer su fuerza / poder, emanados de las relaciones sociales. En el caso de los alumnos de la disciplina de enseñanza de matemáticas, existía una relación de poder, en que el estudiante era el dominado y el profesor el dominante, y para ellos esa relación, quedaba más evidente en el momento de la realización de las evaluaciones.

Pero para la profesora, la relación era de igualdad, diálogo y concesión, pero en ningún momento, ella cedió la propuesta a los estudiantes de no realizar el examen, sin embargo, para la profesora, faltaron argumentos para que los alumnos cuestionaran con éxito el uso del examen.

La profesora entiende los sentimientos de los estudiantes, pero refuerza que ese instrumento de evaluación, además de formativo, sirve para que en el futuro ellos entienden la importancia de evaluar. Ella quería dejar claro que ellos deberían ser "sometidos como alumnos a la disciplina que deberían como profesores imponer más tarde". (FOUCAULT, 1987, p. 322). El cuadro 1 relaciona algunos sentidos que la evaluación, especialmente el examen, tiene para la profesora y para los estudiantes, a partir del análisis de los discursos de las observaciones provenientes de las herramientas de investigación.

Tabla 1. Sentidos de evaluación - el Examen.

\begin{tabular}{|c|c|c|}
\hline \multicolumn{2}{|c|}{ El examen en la disciplina de enseñanza de matemáticas del Curso de Pedagogía de una IES pública del } \\
estado de Ceará - semestre 2017.1
\end{tabular}

Fuente: Elaboración propia. 


\section{RECIMA21 - REVISTA CIENTÍFICA MULTIDISCIPLINAR ISSN 2675-6218}

REFLEXIONES DE UNA PROFESORA DE ENSEÑANZA DE MATEMÁTICAS SOBRE LOS INSTRUMENTOS DE EVALUACIÓN Maria Jose Costa dos Santos, Vladiana Costa dos Santos

El cuadro 1 nos permite volver a la pregunta: ¿Cuál es la relación de los estudiantes de la disciplina de enseñanza de matemáticas con el sentido de evaluar? A partir de lo que nos presenta el cuadro 1, entendemos que para el alumno, cuando el profesor usa el examen, en realidad quiere castigarlo. En este sentido, estamos ante una gran problemática, y hay que pensar en la transformación de las acciones pedagógicas de forma colectiva, buscando subsidios para el conocimiento y el combate a las contradicciones de las prácticas en el aula, especialmente, por estos análisis representar el escenario de un curso de formación pedagógica.

Se trata de mantener la imparcialidad, pero durante nuestras observaciones, en el aula, no percibimos que el examen fuese para "castigar" a los estudiantes. Notamos que la profesora se preocupaba por el proceso, y su enfoque era pautado en una evaluación diagnóstica y formativa, en un proceso continuum, buscando la evaluación como estrategia para reinversión en el 'error'. Ella, después de cada evaluación, comentaba en el aula, y conversaba particularmente con los estudiantes que no habían obtenido resultado positivo, y algunas veces pedía que éstos hicieran de nuevo el examen.

La subjetividad de la práctica y de los sentidos empleados en los discursos de los estudiantes y profesora, no nos permite apuntar un camino. Resaltamos que para nosotros sea cual sea el instrumento utilizado para evaluar, lo importante es lo que se pretende con él. En ese contexto dejamos la reflexión: ¿Para qué sirve el examen en los cursos de formación docente?

\section{CONSIDERACIONES}

En el caso de que se produzca un cambio en la calidad de la información, se debe tener en cuenta que para ello, alertamos a los involucrados con el proceso de evaluación en la escuela, que si quiere mantener consistencia epistemológica y credibilidad de la práctica pedagógica, debe mantener la cohesión entre concepción y práctica.

En este escenario, es importante que el profesor escuche a los estudiantes incluidos en el proyecto pedagógico que busca no sólo el resultado, sino la construcción del proceso con foco en la calidad de la educación. También escuchar a los alumnos, ayuda en la adecuación de los instrumentos evaluativos a las necesidades de ellos, y los motiva a adaptarse al proceso, pues pasan a sentirse parte y no se les es impuesto y sí presentado de forma dialéctica.

En nuestros análisis, percibimos que aunque la profesora buscaba ser la más democrática posible, identificamos indicios de que la evaluación del aprendizaje en el aula era de hecho controlada, y el alumno era sometido al proceso. Esto refleja un poder tan sutil que ni el profesor percibe, pero que la disciplina "define cómo se puede tener dominio sobre el cuerpo de los demás, no simplemente para que hagan lo que se quiere, sino para que operen como se quiere, con las técnicas, según la rapidez y la eficacia que se determina". (FOUCAULT, 1987, p. 164). 


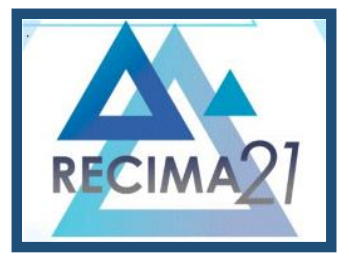

\section{RECIMA21 - REVISTA CIENTÍFICA MULTIDISCIPLINAR ISSN 2675-6218}

Para nosotros, la evaluación del aprendizaje debe romper con la idea de evaluar para castigar o premiar, y debe ser un momento privilegiado de estudio, en el que no sólo el profesor selecciona los contenidos a tratar, sino que el estudiante también sugiere, no importa el instrumento, lo más importante es el tipo de conocimiento que se pone a prueba, el tipo de preguntas que se formulan, el tipo de calidad (mental o práctica) que se requiere y las respuestas que se espera obtener de acuerdo con el contenido de las preguntas o problemas que se hacen, para cualquiera que sea el instrumento utilizado, ya sea el examen, ya sea un seminario, o un taller, lo importante es respetar el proceso, las condiciones de logro y la realidad del estudiante.

Por último, confirmamos que las discusiones aquí presentadas son apenas el preanuncio de las cuestiones que involucran ese tema tan complejo, tanto en el ámbito escolar o en la academia. Así, esperamos en otro momento ampliar y profundizar esa temática, buscando presentar otras experiencias de evaluaciones en el aula, en la academia.

\section{REFERENCIAS}

BALL, D. L. Uncovering the Special Mathematical Work of Teaching. In: KAISER, G. (Eds). Proceedings of the 13th International Congress on Mathematical Education. ICME-13 Monographs. Springer, Cham. 2017.

BRASIL. Lei n.o 9.394, de 20 de dezembro de 1996. Estabelece as Diretrizes e Bases da Educação Nacional. Diário Oficial da União, Brasília, 23 de dezembro de 1996. Disponível em: http://www.planalto.gov.br/ccivil 03/Leis/L9394.htm. Acesso em: 04 maio 2017.

BRASIL. Secretaria de Educação Fundamental. Parâmetro Curricular Nacional: matemática. Brasília: MEC/SEF, 1997.

FERNANDES, D. Para a teoria da avaliação formativa. Revista portuguesa de educação, Portugal, p. 21-50, 2006.

FERREIRA, G. N. L. (Orgs.). A aprendizagem como razão de ensino: por uma diversidade de sentidos. Fortaleza: Imprece, 2016. p. 129-144.

FOUCAULT, M. A Arqueologia do Saber. Tradução de Luiz Felipe Baeta Neves. Rio de Janeiro: Forense Universitária, 2013. 254 p.

FOUCAULT, M. A ordem do discurso: aula inaugural no Collège de France. Tradução de Laura Fraga de Almeida Sampaio. São Paulo: Edições Loyola, 1996.

FOUCAULT, M. Microfísica do poder. Tradução de Roberto Machado. Rio de Janeiro: Edições Graal, 2011. 295 p.

FOUCAULT, M. Vigiar e punir: nascimento da prisão. Tradução de Raquel Ramalhete. Petrópolis: Vozes, 1987. 288p.

FREIRE, P. Pedagogia da autonomia: saberes necessários à prática educativa. São Paulo: Paz e Terra, 1996. (Coleção Leitura)

GIL, A. C. Métodos e técnicas de pesquisa social. São Paulo: Atlas, 1999. 


\section{RECIMA21 - REVISTA CIENTÍFICA MULTIDISCIPLINAR ISSN 2675-6218}

REFLEXIONES DE UNA PROFESORA DE ENSEÑANZA DE MATEMÁTICAS SOBRE LOS INSTRUMENTOS DE EVALUACIÓN Maria Jose Costa dos Santos, Vladiana Costa dos Santos

GONÇALVES, A. L.; LARCHERT, J. M. Avaliação da aprendizagem: pedagogia, módulo 4, volume 6 - EAD. Ilhéus, BA: EDITUS, 2011.

GONÇALVES, V. A.; NASCIMENTO, L. E. Avaliação formativa: autorregulação e controle da textualização. Trabalho em linguística aplicada, Campinas, v. 49, p. 241-257, jan./jun. 2010.

LUCKESI, C. C. Avaliação da aprendizagem: mais uma vez. Revista ABC EDUCATIO, n. 46, jun. 2005.

LUCKESI, C. C. A avaliação da aprendizagem escolar: estudos e proposições. 19. ed. São Paulo: Cortez, 2008.

LUCKESI, C. C. O que é mesmo o ato de avaliar a aprendizagem?. Revista Pátio, ano 3, n. 12, fev./abr. 2000.

MÉNDEZ, J. M. A. Avaliar para Conhecer - Examinar para Excluir. Tradução de Magda Schwartzhaupt Chaves. Porto Alegre, RS: Artmed, 2002.

MONEREO, C. (Coord.). PISA como excusa: repensar la evaluación para cambiar la enseñanza. Barcelona: Editorial Graó, 2009. (Con la colaboración de Monique Boekaerts).

MORETTO, V. P. Prova: um momento privilegiado de estudo, não um acerto de contas. 9. ed. Rio de Janeiro: Lamparina, 2010.

OLIVEIRA, M. M. Como fazer pesquisa qualitativa. Petrópolis, RJ: Vozes, 2010.

PERRENOUD, P. Avaliação: da excelência à regulação das aprendizagens - entre duas lógicas. Tradução de Patrícia Chitinni Ramos. Porto Alegre: Artes Médicas Sul, 1998.

SANTOS, M. J. C. D. Reflexões sobre a formação de educadores matemáticos: a metodologia de ensino Sequência Fedathi. Fortaleza: Imprece, 2016.

SANTOS, M. J. C. D. Plano de ensino da Disciplina Ensino de Matemática - 2016. [S. I.: S. n.], 2017. Disponível grupos.ufc.br/publicações. Acesso em: 05 maio 2017.

SANTOS, M. J. C. D.; LIMA, I. P.; BORGES NETO, H. A Sequência Fedathi: Concepções e princípios para uso no ensino de matemática. In.: VII CIBEM. Montevideo, Uruguai, 16 a 20 de setembro 2013.

SANTOS, Maria José Costa dos. A formação do professor de matemática: metodologia sequência fedathi (sf). Revista Lusófona de Educação, [S. I.], v. 38, n. 38, mar. 2018. ISSN 1646-401X. Disponível em: http://revistas.ulusofona.pt/index.php/rleducacao/article/view/6261. Acesso em: 20 nov. 2018.

SANTOS, Maria José Costa dos; MATOS, Fernanda Cintia Costa. A insubordinação criativa na formação contínua do pedagogo para o ensino da matemática: os subalternos falam? REnCiMa, v. $8, \quad$ n. $\quad 4, \quad$ p. $411-30, \quad 2017.42$ Disponível $\quad$ em: http://revistapos.cruzeirodosul.edu.br/index.php/rencima/issue/view/59.

SOUSA, F. E. E. et al. Sequência Fedathi: uma proposta pedagógica para o ensino de matemática e ciências. Fortaleza: UFC, 2013.

VALENTE, W. R. Avaliação em Matemática: história e perspectiva atuais. Campinas, SP: Papirus, 2008. 


\section{RECIMA21 - REVISTA CIENTÍFICA MULTIDISCIPLINAR ISSN 2675-6218}

REFLEXIONES DE UNA PROFESORA DE ENSEÑANZA DE MATEMÁTICAS SOBRE LOS INSTRUMENTOS DE EVALUACIÓN Maria Jose Costa dos Santos, Vladiana Costa dos Santos

VASCONCELLOS, C. dos S. A avaliação: limites e possibilidades - Algumas Aproximações. Revista Aprender Juntos, São Paulo, n. 2, maio/jun. 2007.

ZANON, D. P.; ALTHAUS, M. M. Instrumentos de avaliação na prática pedagógica

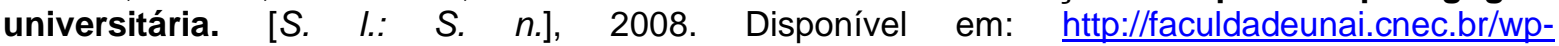
content/uploads/sites/149/2015/10/Instrumentos-de-avaliao-na-prtica-pedaggica-universitria1.pdf. Acesso em: 05 maio 2017. 